\title{
Absence of an association between HLA typing in cystic fibrosis arthritis and hypertrophic osteoarthropathy
}

\author{
Perry J Rush, Dafna D Gladman, Abraham Shore, Karen A B Anhorn
}

\begin{abstract}
Fourteen patients with cystic fibrosis arthritis and eight patients with cystic fibrosis and hypertrophic osteoarthropathy were typed for HLA-A, B, C, DR, and DQ antigens and were compared with age and sex matched controls with cystic fibrosis. The diagnosis of cystic fibrosis arthritis and hypertrophic osteoarthropathy was confirmed by radiography and bone scanning. The prevalence of HLA-A, B, C, D, antigens in the cystic fibrosis group ( 44 patients) did not differ from that in the control group. A comparison between patients with cystic fibrosis arthritis or hypertrophic osteoarthropathy and their respective controls did not show any significant differences in HLA prevalence. It is concluded that HLA antigens may not be a factor in the susceptibility of patients with cystic fibrosis to cystic fibrosis arthritis or hypertrophic osteoarthropathy.
\end{abstract}

Cystic fibrosis is a genetic disease characterised by abnormal secretions from the exocrine glands. In addition to obstructive pulmonary disease and pancreatic insufficiency, cystic fibrosis has also been associated with two distinctly different musculoskeletal diseases - a unique inflammatory polyarthritis (cystic fibrosis arthritis) and classical hypertrophic osteoarthropathy. ${ }^{1}$ As an association between major histocompatibility complex antigens (HLA) and other forms of arthritis has been recognised, ${ }^{2}$ we examined whether the susceptibility to the development of either hypertrophic osteoarthropathy or cystic fibrosis arthritis was linked to HLA system antigens.

\section{Patients and methods}

All patients had clinical and radiological characteristics compatible with cystic fibrosis, including a raised sweat chloride test. The patients were selected from the 560 patients with cystic fibrosis followed up at the Hospital for Sick Children (Toronto, Canada). Fourteen patients met the criteria for cystic fibrosis arthritis characterised by episodic attacks of polyarthritis or a persistent synovitis with progressive asymmetric, erosive disease and had no evidence of any other rheumatic disease. ${ }^{1}$ Fourteen patients with cystic fibrosis but no arthritis who were matched for age, sex, and pulmonary function (assessed by the forced expiratory volume in one second $\left(\mathrm{FEV}_{1}\right)$ ) served as disease controls for cystic fibrosis arthritis. Eight patients had cystic fibrosis and hypertrophic osteoarthropathy, as defined by tenderness along the long bones of the arms or legs and radiographic or bone scan evidence of periosteal elevation at those sites. Eight patients with cystic fibrosis without hypertrophic osteoarthropathy but matched for age, sex, and $\mathrm{FEV}_{1}$ were used as disease controls for hypertrophic osteoarthropathy (table 1).

Pulmonary function testing was measured with a 9 litre water filled Collin's spirometer. The highest value in a series of three forced vital capacity manoeuvres was chosen and the FEV was calculated.

Volunteers from the Canadian Red Cross and hospital staff served as healthy controls for HLA distribution among normal subjects. All patients and controls were white. The microcytotoxicity test of Terasaki $^{2 a}$ was used to identify class I (HLA-A, B, C) and class II (HLA-DR and DQ) antigens. Lymphocytes were separated from peripheral blood by centrifugation over Ficoll-Hypaque gradients. T and B cells were further purified by passage over nylon wool columns. Canadian Red Cross National Reference Laboratory tissue typing trays were used.

Statistical analysis was performed using the $\chi^{2}$ test to compare groups. p Values were corrected by multiplying by the number of antigens tested.

\section{Results}

Table 1 presents the clinical characteristics of the patients tested. The prevalence of HLA-A, $B$, and $C$ in the cystic fibrosis group (44 patients) did not differ from that in normal subjects (data not shown). HLA-DR7 was less common among patients with cystic fibrosis than normal subjects (relative risk $0 \cdot 23$ ) but was

Table 1 Clinical characteristics of patients with cystic fibrosis

\begin{tabular}{llccc}
\hline $\begin{array}{l}\text { Clinical } \\
\text { characteristics }\end{array}$ & Arthritis & No arthritis & $\begin{array}{l}\text { Hypertrophic } \\
\text { osteoarthropathy }\end{array}$ & $\begin{array}{c}\text { No hypertrophic } \\
\text { osteoarthropathy }\end{array}$ \\
\hline Number of patients & 14 & 14 & 8 & 8 \\
Male & 4 & 4 & 6 & 6 \\
Female & 10 & 10 & $25 \cdot 7$ & 2 \\
Mean age (years) & $21 \cdot 5$ & $64 \cdot 5$ & 50 & $25 \cdot 1$ \\
Mean FEV, (\%) & 66 & 64 & & 48 \\
\hline
\end{tabular}


Table 2 Prevalence of class II HLA antigens in patients with cystic fibrosis with arthritis and hypertrophic osteoarthropathy and their cystic fibrosis controls. Results given as number of patients (percentage)

\begin{tabular}{lcccc}
\hline $\begin{array}{l}\text { HLA } \\
\text { antigen }\end{array}$ & $\begin{array}{l}\text { Arthritis } \\
(n=14)\end{array}$ & $\begin{array}{l}\text { No arthritis } \\
(n=14)\end{array}$ & $\begin{array}{l}\text { Hypertrophic } \\
\text { osteoarthropathy } \\
(n=8)\end{array}$ & $\begin{array}{l}\text { No hypertrophic } \\
\text { osteoarthropathy } \\
(n=8)\end{array}$ \\
\hline DR1 & $4(29)$ & $4(29)$ & $1(13)$ & $3(38)$ \\
DR2 & $3(21)$ & $3(21)$ & $3(38)$ & $1(13)$ \\
DR3 & $4(29)$ & $5(36)$ & $1(13)$ & $3(38)$ \\
DR4 & $1(7)$ & $7(50)$ & $3(38)$ & $2(25)$ \\
DR5 & $3(21)$ & 0 & 0 & $1(13)$ \\
DR6 & $3(21)$ & $4(29)$ & $2(25)$ & $3(38)$ \\
DR7 & $2(14)$ & $1(7)$ & 0 & $1(13)$ \\
DR8 & $3(21)$ & 0 & $1(13)$ & 0 \\
DR9 & 0 & $1(7)$ & $4(50)$ & 0 \\
DR10 & $1(7)$ & $7(57)$ & $5(63)$ & $6(75)$ \\
DRw52 & $10(71)$ & $10(71)$ & $4(50)$ & $3(38)$ \\
DRw53 & $2(14)$ & $6(43)$ & 0 & $6(75)$ \\
DQw1 & $8(57)$ & $6(43)$ & $2(25)$ & $3(38)$ \\
DQw2 & $6(43)$ & $5(36)$ & & $4(50)$ \\
DQw3 & & $13)$ & \\
\hline
\end{tabular}

not statistically significant. There were no significant differences in HLA prevalence in class I (results not shown) or class II antigens (table 2) between patients with cystic fibrosis arthritis or hypertrophic osteoarthropathy and their disease controls. There was a lower prevalence of HLADR4 in patients with cystic fibrosis and arthritis compared with the other groups, but this was not statistically significant.

\section{Discussion}

HLA typing in cystic fibrosis has been reported by six different groups from 1973 to 1980 . Five of the six groups reported no differences in the prevalence of HLA-A, B, or C antigens in patients with cystic fibrosis compared with normal subjects. ${ }^{3-7}$ Only Kaiser et al reported an increase in HLA-B18. ${ }^{8}$ We have confirmed the absence of an association between HLA-A, $B$, and $C$ antigens and cystic fibrosis and have extended this observation to include HLA class II antigens. The decreased prevalence of HLADR7 and the low relative risk associated with it $(0.23)$ may suggest that this antigen confers some protection against arthritis. HLA-DR7 was not present in any of the patients with hypertrophic osteoarthropathy. Unlike other forms of childhood arthritis there was no association with HLA-DR4 or HLA-DR5.

The aetiology of cystic fibrosis arthritis is unclear. The major histocompatibility complex has been associated with numerous diseases causing arthritis-for example, HLA-DR4 and rheumatoid arthritis. ${ }^{2}$ In addition, patients with HLA-B27 are susceptible to the development of reactive arthritis after infection of the gastrointestinal or genitourinary tract. ${ }^{9}$ Some of the clinical features of cystic fibrosis arthritis are similar to those of reactive arthritis. ${ }^{1}$ The respiratory and gastrointestinal tracts in patients with cystic fibrosis are both altered not only by the disease but also by long term drug treatment. Changes in the endogenous microflora or enhanced systemic penetration of these organisms as a result of this combination of factors could result in a reactive arthritis in susceptible cystic fibrosis hosts-for example, those with an HLA-B27 haplotype.

We found no increase in HLA-B27 or any other HLA antigen in our patients with cystic fibrosis arthritis. Dixey et $a l,{ }^{10}$ in a clinical study of cystic fibrosis arthritis and hypertrophic osteoarthropathy, reported a normal distribution of class I (A, B, C) HLA antigens in patients with cystic fibrosis arthritis or cystic fibrosis with hypertrophic osteoarthropathy compared with patients with cystic fibrosis without arthritis or normal subjects. However, their report did not present any HLA data and did not state how many patients were typed. No disease controls with cystic fibrosis were used.

The pathogenesis of hypertrophic osteoarthropathy is unknown. Primary hypertrophic osteoarthropathy probably has a hereditary component as there is a familial predisposition. No HLA typing has been reported previously in patients with secondary hypertrophic osteoarthropathy. We have shown that in patients with hypertrophic osteoarthropathy secondary to cystic fibrosis there is no HLA association.

This work was partially supported by the Arthritis Society.

1 Rush P J, Shore A, Coblentz C, et al. The musculoskeletal manifestations of cystic fibrosis. Semin Arthritis Rheum 1986; 15: 213-25.

2 Gladman DD, Anhorn K A B. HLA and disease manifestations in rheumatoid arthritis-a Canadian experience. 7 R heumatol in rheumatoid ar

2a Terasaki P I, Bernoco D, Park M S, Ozturk G, Iwaki Y. Microdroplet testing for HLA-A, -B, -C, and -D antigens. Am $\mathcal{F}$ Clin Pathol 1978; 69: 103-20.

3 Goodchild M C, Edwards J H, Glenn K P, et al. A search for linkage in cystic fibrosis. F Med Genet 1976; 13: 417-9.

4 Hennecquet $\mathrm{A}$, Jehanne $\mathrm{M}$, Betuel $\mathrm{H}$, et al. Cystic fibrosis and HLA. Tissue Antigens 1978; 12: 159-62.

5 Polymenidis $Z$, Ludwig H, Gotz M. Cystic fibrosis and HLA antigens. Lancet 1973; ii: 1452 .

6 Tobin M J, Maguire O, Reen D, et al. Atopy and bronchial reactivity in older patients with cystic fibrosis. Thorax 1980 35: 807-13.

7 Safwenberg J, Kollberg H, Lindblom J. HLA frequencies in patients with cystic fibrosis. Tissue Antigens 1977; 10: 287-90 Kaiser G I, Laszlo A, Gyurkovits K. Cystic fibrosis: a HLA associated hereditary disease? Acta Paediatr Hung 1977; 18 :
27-9.

9 Brewerton D A, Caffrey M, Nicholls A. Reiter's disease and HLA27. Lancet 1973; ii: 996-8.

10 Dixey J, Redington AN, Butler RC, et al. The arthropathy of cystic fibrosis. Ann Rheum Dis 1988; 47: 218-23. 